\title{
Encoding, consolidation, and retrieval of contextual memory: Differential involvement of dorsal CA3 and CAl hippocampal subregions
}

\author{
Stéphanie Daumas, ${ }^{1}$ Hélène Halley, Bernard Francés, and Jean-Michel Lassalle ${ }^{2}$ \\ Centre de Recherches sur la Cognition Animale, Centre National de la Recherche Scientifique UMR 5169, Université Paul \\ Sabatier, Toulouse, France
}

\begin{abstract}
Studies on human and animals shed light on the unique hippocampus contributions to relational memory. However, the particular role of each hippocampal subregion in memory processing is still not clear. Hippocampal computational models and theories have emphasized a unique function in memory for each hippocampal subregion, with the CA3 area acting as an autoassociative memory network and the CA1 area as a critical output structure. In order to understand the respective roles of the CA3- and CA1-hippocampal areas in the formation of contextual memory, we studied the effects of the reversible inactivation by lidocaine of the CA3 or CA1 areas of the dorsal hippocampus on acquisition, consolidation, and retrieval of a contextual fear conditioning. Whereas infusions of lidocaine never impaired elementary tone conditioning, their effects on contextual conditioning provided interesting clues about the role of these two hippocampal regions. They demonstrated first that the CA3 area is necessary for the rapid elaboration of a unified representation of the context. Secondly, they suggested that the CAl area is rather involved in the consolidation process of contextual memory. Third, they showed that CAl or CA3 inactivation during retention test has no effect on contextual fear retrieval when a recognition memory procedure is used. In conclusion, our findings point as evidence that CAl and CA3 subregions of the dorsal hippocampus play important and different roles in the acquisition and consolidation of contextual fear memory, whereas they are not required for context recognition.
\end{abstract}

Studies in higher primates and humans have led to the idea that the hippocampus is required for different types of memory, such as declarative (Squire 1992) or episodic memory (Tulving 1983). Independently of the type of memory, the hippocampus might be engaged in different memory processes, such as encoding, consolidation, and retrieval. Lesion studies in animals provide further support for the transient requirement of the hippocampus in the process of long-term memory formation (Kim and Fanselow 1992; Anagnostaras et al. 1999) in agreement with the theory of Squire (1992), describing the hippocampus as a temporary memory buffer that enlists the prefrontal cortex, where the information is ultimately stored.

The hippocampal structure is functionally heterogeneous, with different portions of the longitudinal axis having different functional roles, certainly due to differences in connectivity (Moser and Moser 1998). Indeed, the dorsal hippocampus seems to be highly involved in spatial learning (Moser et al. 1993, 1995), which is consistent with the major visuo-spatial inputs received from the temporal and parietal cortices, whereas the ventral hippocampus presents a strong connectivity with both the hypothalamus and the amygdala, which potentially accounts for some effects of the hippocampal lesion on emotionality (Kjelstrup et al. 2002). Moreover, all hippocampal subregions are highly interconnected, and their arrangement suggests that, individually, they may subserve discrete computational functions.

\footnotetext{
1Present address: Division of Neuroscience, School of Biomedical \& Clinical Laboratory Sciences, University of Edinburgh, 8 Crichton Street, EH8 9LE, Edinburgh, UK.

${ }^{2}$ Corresponding author.

E-mail lassalle@cict.fr; fax 33-561-556-154.

Article published online ahead of print. Article and publication date are at http://www.learnmem.org/cgi/doi/10.1101//m.81905.
}

The uncommon neuronal architecture of the CA3 region allows local associative synaptic modification, recurrent activation, and sparse random activity to occur, suggesting that it might serve as an autoassociative memory, where multimodal information could be processed as an integrated representation, then stored and ultimately completely retrieved from partial or degraded inputs (Nakazawa et al. 2002). Therefore, the CA3 network has been allocated the capacity to enable rapid acquisition of unique associations and to store patterned information received from the dentate gyrus or directly from the entorhinal cortex for a short period of time (Rolls and Treves 1996). Accordingly, we can hypothesize a crucial role of the CA3 network in contextual memory acquisition. Such autoassociative properties are not found in the CA1 area, considered as the major output structure of the hippocampus. Nevertheless, an information-processing function has also been assigned to this subregion. Indeed, the CA1 area might be instrumental in recognizing the novelty or familiarity of an object or context (see Nakazawa et al. 2004). Therefore, this area might be rather involved in the consolidation and retrieval of recent contextual memory, than in its processing.

According to numerous theories on the functional differentiation of hippocampal areas, some recent studies have tried to better understand the specific roles of these two hippocampal subregions in learning and memory. They have focused on the study of neurotransmitter receptors (Tsien et al. 1996; Riedel et al. 2000; Lee and Kesner 2002; Nakazawa et al. 2002) or on the understanding of intrahippocampal connections (Brun et al. 2002). Nevertheless, most studies that have focused on hippocampal subregions involved only irreversible lesions, which does not allow a clear understanding of differential involvement of CA1 and CA3 areas in learning and memory processing (Lee and Kesner 2004a,b; Lee et al. 2004). 
The purpose of this study was to shed light on the respective involvements of the CA3 and CA1 areas in the different stages of contextual memory processing in mice, using focused and reversible lesions.

In classical fear conditioning in rodents, pairing a conditional stimulus (CS) with an aversive unconditioned stimulus (US), such as an electric foot-shock, elicits a conditioned fear response, such as freezing (Phillips and LeDoux 1992). Although the CS can be a tone, as well as the context, the resulting learning process is different in each case. On the one hand, tone-US association is based on a simple associative learning. On the other hand, taking the context as a CS entails relational learning to form a unified representation of environmental stimuli and their mutual relations, a process that may contribute to form a configural representation and also participate in the formation of human declarative memory (Eichenbaum 1996). In other words, whereas auditory fear conditioning involves discrete unisensory information processing, contextual fear conditioning, in contrast, involves multisensory information processing of present stimuli. For instance, episodic memory stores a kind of relational representation that provides information not only about the "what" of events, but also about the "where" and the "when" they occurred. Relational learning has been linked to processes underlying the formation of spatial and episodic memory and, in contrast to simple associative learning, has been associated with the hippocampus (Eichenbaum 1996; Anagnostaras et al. 2001; Kandel 2001; Morris 2001).

In order to investigate information processing within the CA3 and CA1 areas of the dorsal hippocampus, we chose a reversible inactivation procedure, using lidocaine microinfusions. Lidocaine is a local anesthetic agent that produces reversible inactivation of neural tissue via blockade of voltage-gated sodium channels. Infusions were made either before or immediately after conditioning to focus on encoding or consolidation memory processes, or before the memory test, to act specifically on retrieval.

\section{Results}

\section{Histology}

After histological examination of brain slices, 18 mice of a total of 111 have been taken out of the analyses because of unilateral infusion occurrence of the drug or of misplacement of guide cannulae and/or injection. In all remaining mice, the tips of the infusion cannulae were located in the hippocampal area of interest (see Fig. 1).

\section{Experiment 1: Time- and region-dependent effects of pre-conditioning lidocaine infusion in the dorsal hippocampus}

\section{Locomotor activity}

First of all, in order to check the risk of a side effect of the lidocaine infusion on locomotion, the general activity of mice was assessed by counting the number of experimental chamber crossings during the first $2 \mathrm{~min}$ of conditioning. The ANOVA analysis revealed no significant overall variation of locomotor activity among the different groups treated before conditioning $\left[F_{(3,28)}=1.07, P=0.378\right]$, as can be seen from Figure 2 .

\section{Time efficiency and reversibility of lidocaine}

In order to know precisely how long lidocaine remains active in the hippocampus and to ascertain its good functional reversibility, two groups of mice were injected with lidocaine in the CA3
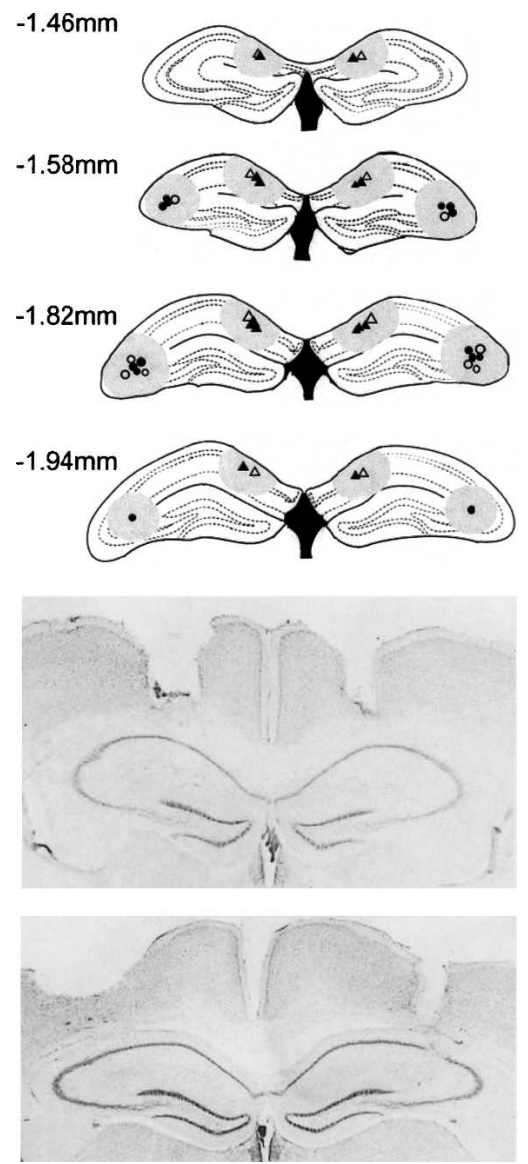

Figure 1. Location of intrahippocampal infusion sites, example from pre-conditioning injected mice. The distribution of cannulae placements is figured for Control (open shape) and Lidocaine-treated animals (solid shape) for all CA3- (circles) and CA1-injected mice (triangles). The maximum spread of the drug within the tissue is indicated by grayed circles (assessed by a dye experiment). Numbers beside each section indicate the distance in $\mathrm{mm}$ posterior to Bregma. Pictures show the guide cannulae tracks for CA1 (above) and CA3 (below) infusions.

area, either 10 or 15 min before conditioning (Fig. 3). The ANOVA revealed a significant variation of freezing values among injected groups $\left[F_{(2,22)}=9.991, P=0.001\right]$. Post-hoc comparisons indicated significantly lower freezing values in lidocaine-injected mice after a 10 -min delay $(P=0.001)$, but not after a 15 -min delay $(P=0.622)$, than in NaCl-injected mice. Consequently, in further experiments, lidocaine was injected $10 \mathrm{~min}$ before conditioning.

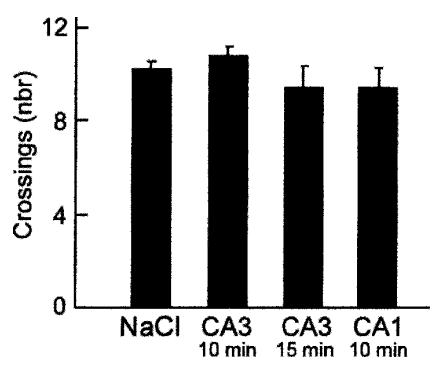

Figure 2. Locomotor activity of mice infused with lidocaine 15 or 10 min prior to conditioning (Experiment 1). Locomotor activity is represented by the total number of chamber crossings during the first 2 min of the conditioning, before the first electric foot-shock. No variation between groups can be observed $(P=0.378)$. 


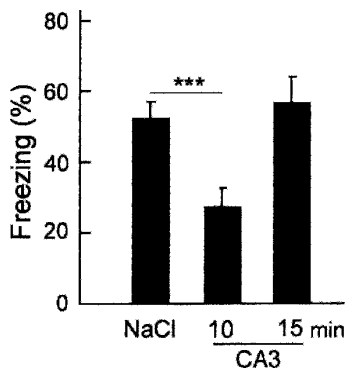

Figure 3. Time efficiency of lidocaine injected in the CA3 region. Results are presented as the percentage of time spent freezing during the contextual test (mean \pm SEM). Lidocaine infused in the CA3, 15 min before conditioning, failed to impair the contextual fear acquisition in regard to $\mathrm{NaCl}$ control $(P=0.622)$, whereas infusion of lidocaine $10 \mathrm{~min}$ before conditioning impairs significantly contextual fear conditioning $(* * *) P=0.001$ vs. $\mathrm{NaCl}$.

\section{Short-term memory and conditioning}

Injecting mice before conditioning allows us to act on the first stage of memory processing, i.e., the acquisition or encoding phase. Although the absolute level of the fear response could be influenced by many factors, including strain-specific shock sensitivity and reaction to stress hormones, freezing reactions emerging after the first shock during the conditioning session are considered as a conditioned fear response depending on shortterm memory (Kim et al. 1992; Fanselow 2000; Anagnostaras et al. 2001). Data presented in Figure 4 show the evolution of freezing during the 2-min period after the shock. They reflect the rapid acquisition of conditioned fear immediately after the first shock. Although the repeated factor analysis of variance showed a marginal group effect $\left[F_{(2,21)}=3.093, P=0.066\right]$ with no time effect $\left[F_{(2,42)}=2.433, P=0.10\right]$ and a significant groups $\times$ time interaction $\left[F_{(4,42)}=2.811, P=0.037\right]$ a planned comparison revealed no difference between CA3- and CA1-treated mice $\left[F_{(1,14)}=0.304, P=0.59\right]$. In contrast, a significant difference appeared between the lidocaine-treated groups (CA3 and CA1) and the control group $(\mathrm{NaCl})\left[F_{(1,22)}=5.957, P=0.023\right]$.

\section{CA3- and CA1-area-specific lidocaine disruption and contextual}

\section{fear acquisition}

A significant variation in freezing levels between groups $\left[F_{(2,22)}=5.983, P=0.008\right]$, was observed during retrieval, $24 \mathrm{~h}$

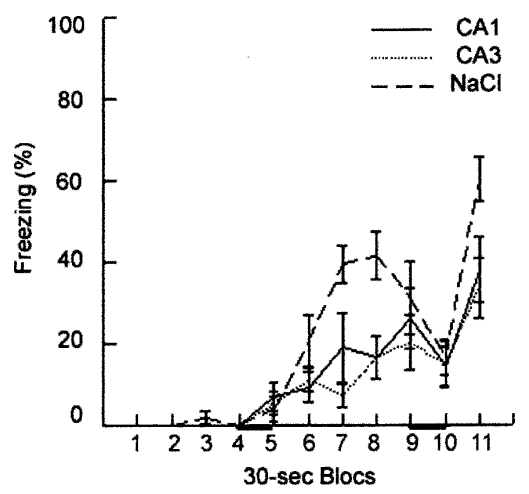

Figure 4. Percentage of time spent freezing during conditioning (mean \pm SEM). The 30 -sec sound emission is represented by a bold line on the $x$-axis. Freezing behavior occurring just after the first shock is accepted to reflect short-term memory. During the three 30-sec blocks following the shock, we observe that fear expression increased less in the two groups of mice treated with lidocaine 10 min before conditioning than in the $\mathrm{NaCl}$-injected group $(P=0.023)$. after injection, (Fig. 5A). Post-hoc comparisons revealed a significant effect of the treatment made either in the CA3 or in the CA1 subregion [respectively, $P=0.009$ and $P=0.006$ vs. NaCl]. No difference appeared between CA3- and CA1-injected mice $(P=0.686)$. Group comparisons of freezing levels expressed during the first and second minute of the contextual test (Fig. 5B) showed a significant increase for $\mathrm{NaCl}$ - and CA3-treated groups [respectively, $F_{(1,8)}=11.011, P=0.011$ and $F_{(1,8)}=9.434$, $P=0.015]$ but not for the CA1 group $\left[F_{(1,6)}=1.691, P=0.241\right]$. Therefore, whereas lidocaine injection in the CA1 or in the CA3 area impaired contextual learning, only CA1 infusion clearly disrupted the expectancy of a frightening event $2 \mathrm{~min}$ after the reintroduction in the conditioning chamber. Freezing to the tone (Fig. 5C) was not disrupted by any local infusion of lidocaine in the dorsal hippocampus $\left[F_{(2,22)}=1.471, P=0.251\right]$.

\section{Experiment 2: Region-dependent effects of post-conditioning lidocaine infusion in the dorsal hippocampus}

\section{CA3- and CA1-area-specific lidocaine disruption and contextual fear consolidation}

In this second experiment, lidocaine has been injected immediately after conditioning. As can be seen from Figure 6A, this resulted in a significant overall variation of contextual fear $\left[F_{(2,21)}=10.977, P=0.001\right]$, with CA1 lidocaine-injected mice expressing significantly lower freezing than those injected in the CA3 subregion $(P=0.013)$ that, in turn, displayed less freezing than NaCl-treated mice $(P=0.04)$. Comparing the evolution of freezing levels between the different groups during the first $2 \mathrm{~min}$ (Fig. 6B) revealed a significant increase of freezing in the $\mathrm{NaCl}-$ $\left[F_{(1,7)}=5.494, P=0.05\right]$, but not in the CA1- and CA3-injected groups [respectively, $F_{(1,6)}=0.001, P=0.976$ and $F_{(1,8)}=0.179$, $P=0.684]$. When analyzing the evolution of freezing scores in the different groups over the four time intervals, a significant variation of freezing kinetics appeared with time for the $\mathrm{NaCl}$ and CA3-injected groups [respectively, $F_{(3,21)}=3.156, P=0.047$ and $\left.F_{(3,24)}=3.042, P=0.048\right]$, that was not apparent in the CA1injected group $\left[F_{(3,18)}=0.646, P=0.596\right]$. This consequence of lidocaine injection after conditioning in the CA1 area is reminiscent of what we obtained with pre-conditioning injections (Fig. 5) and suggests a major role of the CA1 dorsal hippocampus in the temporal component, the "when" of episodic memory. This temporal effect linked to the event expectancy was fading proportionally to the impairment of the memory of the context. Freezing to the tone (Fig. 6C) was not disrupted by any local microinfusion of lidocaine in the dorsal hippocampus $\left[F_{(2,21)}=2.897, P=0.077\right]$.

In order to better understand the consequences of hippocampal disruptions induced by lidocaine infusion in the CA1 or CA3 area, the effects of pre- and post-conditioning injections of lidocaine were compared (Fig. 7). The ANOVA revealed a significant effect of the moment of injection for the CA1 group $\left[F_{(1,12)}=5.309, P=0.04\right]$ not observed in case of CA3 inactivation $\left[F_{(1,16)}=2.002, P=0.176\right]$. This comparison allows us to propose that CA1 plays a more important role in consolidation than CA3.

\section{Experiment 3: Effects of pre-test lidocaine injection} in the dorsal hippocampus on contextual memory retrieval

\section{No effect of CA3- and CAI disruption by lidocaine on contextual fear retrieval}

Infusions of lidocaine in the CA1 or CA3 area before the memory test had no consequence on contextual memory retrieval (Fig. $8 \mathrm{~A}),\left[F_{(2,23)}=1.383, P=0.271\right]$. The comparison of freezing bouts 


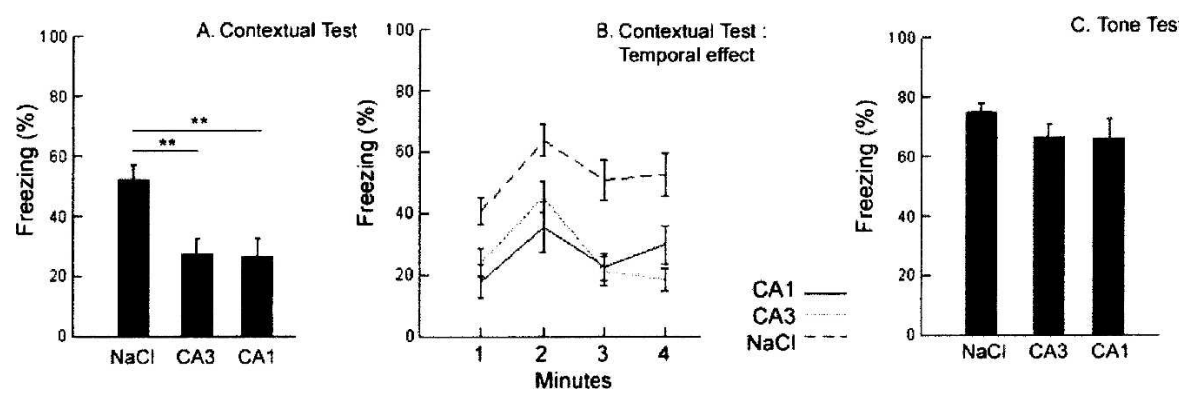

Figure 5. Effects of $C A 3$ and CA1 pre-conditioning infusions of lidocaine on contextual and tone fear conditioning (Experiment 1). ( $A$ ) Contextual fear; CA1 pre-conditioning disruption induced a diminution of contextual fear conditioning $\left(^{* *}\right) P=0.006$ in the same range as CA3 disruption $\left({ }^{* *}\right) P=0.009$. $(B)$ Kinetics of contextual freezing during the contextual test. (C) No significant effect of preconditioning drug infusion is revealed by the conditioning to the tone $(P=0.251)$.

expressed during the first $2 \mathrm{~min}$ revealed a significant increase in each experimental group $\left[\mathrm{NaCl}, F_{(1,8)}=6.291, P=0.036\right.$; CA3, $F_{(1,8)}=9.377, P=0.016$, and CA1, $\left.F_{(1,7)}=5.846, P=0.046\right]$, indicating that mice expressed similar temporal expectancy of the event as illustrated in Figure 8B. Figure 8C shows that no inactivation impaired tone conditioning $\left[F_{(2,23)}=0.167, P=0.847\right]$.

\section{Discussion}

Based on focused reversible inactivations that allow dissociating the different stages of memory processing, these experiments yielded three major findings. First, the dorsal CA1 area, but also the CA3 network, is essential for an optimal acquisition of contextual memory. Secondly, these two hippocampal subregions are clearly involved in contextual memory consolidation, although CA1 inactivation leads to greater disruption of contextual memory. Third, surprisingly, dorsal CA1 and CA3 areas are not necessary for contextual retrieval. Moreover, as could be predicted from the literature, tone conditioning was not affected by any treatment. Finally, the kinetics of freezing behavior was clearly disturbed after post-conditioning lidocaine infusions in the CA1 area, suggesting a possible implication of this area in the memory processing of "when" the event occurred.

\section{Lidocaine as a tool to study the role of hippocampal regions in contextual memory}

Since neurotoxic and excitotoxic hippocampal lesions often result in locomotor disturbances (Anagnostaras et al. 1999; Zhang et al. 2002; Bast and Feldon 2003), we first examined the consequences of lidocaine infusions in the CA3 or CA1 area on locomotor activity during the first 2 min of the conditioning protocol, before US occurrence. Locomotion, measured by the number of chamber crossings, was not affected by microinjections of lidocaine, indicating that resulting contextual memory impairments were not due to a side effect of the drug on locomotion.

Mice injected with lidocaine 15 min before the learning session performed as well as the $\mathrm{NaCl}$ control group, whereas after a shorter delay of $10 \mathrm{~min}$, they were impaired. These results are in accordance with the existing literature on the short duration of action of lidocaine (Martin 1991; Tehovnik and Sommer 1997). They demonstrate in our
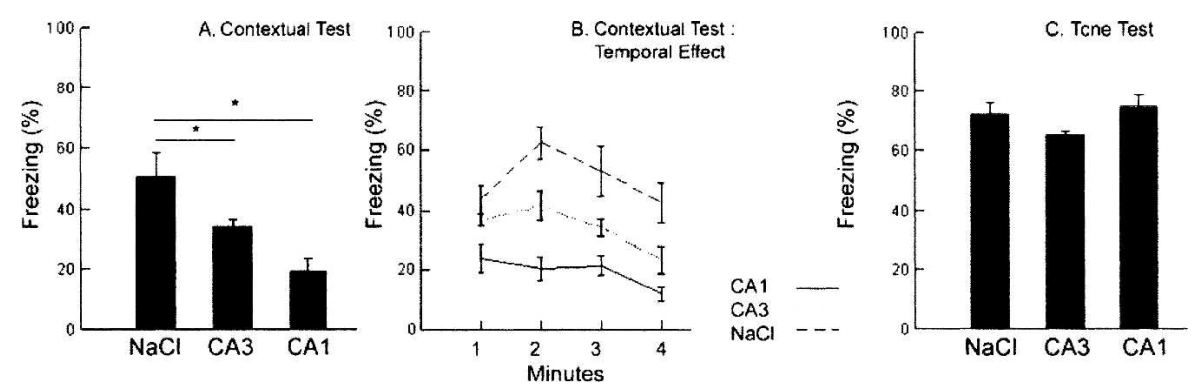

Figure 6. Effects of post-conditioning infusions of lidocaine on Contextual fear memory consolidation (Experiment 2). (A) Contextual fear; CA3 disruption induces a significant decrease of the contextual freezing level; $\left(^{*}\right) P=0.04$. Hippocampal CA1 infusion leads to a greater impairment of contextual consolidation than CA3 infusion; $\left(^{*}\right) P=0.013$. (B) Freezing kinetics during the contextual test. Postconditioning CA1 lidocaine infusion abolishes event expectation, a particular feature of episodic memory. (C) No significant effect of post-conditioning drug infusion is revealed in the tone conditioning; $(P=0.077) .\left(^{*}\right) P<0.05,\left({ }^{* *}\right) P<0.01$, $\left({ }^{* * *}\right) P<0.001$. 

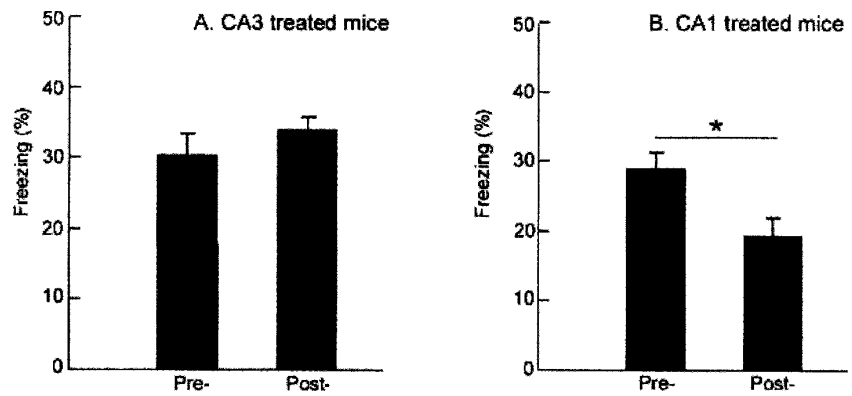

Figure 7. Differential effects of site specific CA3- $(A)$ and CA1- (B) lidocaine infusions on acquisition and consolidation levels of contextual fear. Mice injected in the CA3 area before or after conditioning show no conditional fear difference, whereas conditional levels of CA1-injected groups differ. Mice injected in the CA1 area after conditioning show a greater impairment than mice infused before conditioning $\left({ }^{*}\right), P=0.04$, indicating that the CA1 area may play a more important role in contextual memory consolidation.

more generally, the hippocampus, may not be the only structure that can process complex representations. Accordingly, it can be suggested that hippocampo-independent surrogate strategies might be implemented by mice in order to associate to the footshock a features representation of the context, where the context is represented as a set of independent features or elements, each of them liable to enter into an association with the shock (Rudy and O'Reilly 2001). Anyhow, the aim of our study was less to demonstrate complete impairment of the contextual fear conditioning than to compare the consequences of lesions of the same range in different hippocampal subregions.

Following a pre-conditioning microinfusion of lidocaine, fear memory to the context, but not to the tone, was impaired in both treated groups. This result suggests that both areas are involved in the acquisition of contextual fear memory, the CA3 area, certainly through its involvement in the rapid formation of a unified representation of the context, and the CA1 area by its strategic place as a critical output structure, leading to the conclusion that contextual information is rapidly processed in the autoassociative CA3 network, then sent to the CA1 to be stored ultimately in the neocortex. These conclusions are strengthened by previous work showing that CA3-NMDA receptors are crucial for rapid hippocampal encoding of unique events (Nakazawa et al. 2003).

\section{CA1 vs. CA3 implication in the consolidation of contextual fear memory}

In our study, disruption of the CA3 network immediately after conditioning impaired contextual fear memory less than CA1 disruption. As lidocaine completely abolishes electric events by blocking voltage-dependant sodium channels, and subsequently, neurotransmission and LTP, it will result in an interruption of the early consolidation process. Nevertheless, there might be enough time between the first electric-shock and lidocaine infusion for consolidation to be initiated, which would explain residual freezing observed in both injected groups.

All together, it appears that CA3 activity is necessary for contextual memory consolidation, but also that CA1 activity after fear conditioning has an even larger involvement. Our results match previous work showing that NMDA receptors, $\beta$-adrenoreceptors, but also metabotropic glutamate receptors in CA1 would play a crucial role in spatial and/or contextual learning abilities, namely in the consolidation process (Riedel et al. 2000; Shimizu et al. 2000; Nakazawa et al. 2002; Ji et al. 2003).

Moreover, we have found a clear impairment of the tempo- ral evolution of freezing in the CA1-treated group, suggesting that the CA1 area might be involved in temporal information processing. The results of Huerta et al. (2000), obtained from knockout mice lacking NMDARs only in hippocampal CA1 pyramidal cells, also suggest that the CA1 area is crucial for the formation of memories that associate events over time.

\section{Contextual retrieval and hippocampal CA3 and $\mathrm{CAl}$ areas}

The hippocampus subsequently plays a time-limited role in memory by enabling the gradual development of intracortical connections that render cortical memory traces enduring and self-sufficient, a process that Morris et al. (2003) referred to as systems-level consolidation (Squire 1992; McClelland et al. 1995). Hippocampal neuronal activity is generally acknowledged as being involved in the retrieval of recent episodic memories, whereas it is not for more remote memories (Kim and Fanselow 1992). However, our results did not show any disruption of contextual fear retrieval following inactivation of dorsal hippocampal CA1 or CA3 subregions during the contextual memory test. This outcome could imply that memory trace retrieval, $24 \mathrm{~h}$ after learning, is already independent of these hippocampal subregions. For instance, Fortin et al. (2002), using radiofrequency lesions of the entire structure of rats, demonstrated that the hippocampus is essential to learn a sequence of events, but is not required to recognize items that occur in a unique series of events. Therefore, it is possible that a functional hippocampus is needed for the recall of only some types of memories, and that in the case of contextual fear memory, independent activation of CA1 and CA3 subregions is not required for retrieval, this role being then devoted to some other cortical areas. Nevertheless, it has also been demonstrated that (1) lesions made $1 \mathrm{~d}$ after conditioning resulted in a large contextual memory impairment (Kim and Fanselow 1992), and that (2) the CA3 network can support a recall mechanism named pattern completion (Nakazawa et al. 2002), which could be involved in some instances of contextual fear recall (Rudy and O'Reilly 2001). Thus, the CA1 and CA3 subregions seem to be necessary for memory retrieval. To account for our discrepant results, it could be objected that the microinjections performed in our study lead to restrained inactivation that would not allow revealing the involvement of these two areas in contextual memory retrieval. The important result from our study remains, nonetheless, that same-range disruptions of hippocampal CA1 and CA3 subregions result in a deficit in the encoding and consolidation of memory, whereas they spare the retrieval mechanism, leading to the observation that hippocampal networks are not evenly requested during all stages of contextual memory processing. A more compelling explanation can nevertheless be put forward; retrieval tests in animal experiments generally make use of recognition rather than recall procedures, as is the case in our study, so that the subject can recognize the environment, which does not need a functional hippocampus, since it has been widely demonstrated in humans and animals that recognition memory remains relatively performing when the hippocampus has been damaged either by ischemia (Mayes et al. 2004), or lesions of various origins (Squire et al. 2001), experimental lesions (Forwood et al. 2005), aging (McIntosh et al. 1999; Sekuler et al. 2005), or even degenerative processes as in Alzheimer's disease (Karlsson et al. 2003).

\section{Conclusions}

Our results demonstrate clearly that both the CA3 and CA1 subregions of the dorsal hippocampus play important and complementary roles in the encoding and consolidation processes of contextual memory. In contrast, contextual retrieval can occur 

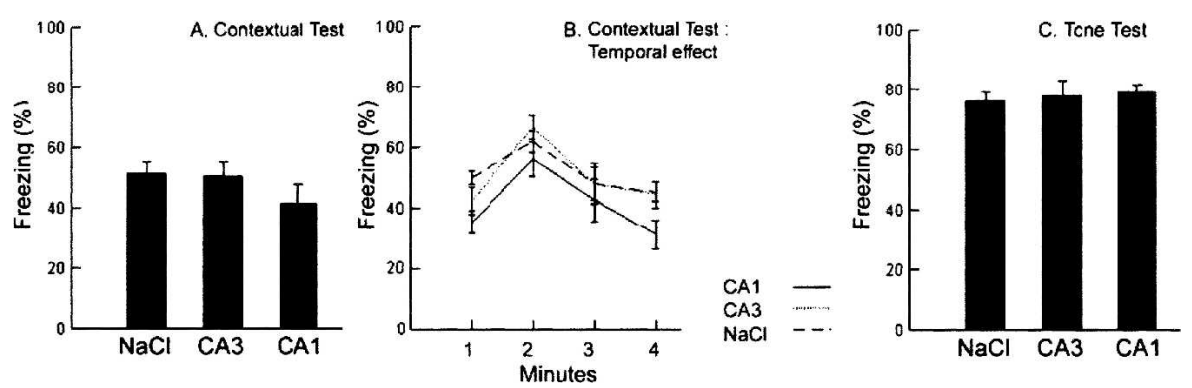

pon, Bayer Pharma). Stainless-steel guide cannulae $(24 \mathrm{G})$ were implanted bilaterally in the cortex above the dorsal hippocampus using standard stereotaxic procedures. Cannulae coordinates for the CA1 infusion site were as follows: (AP) $-1.6 \mathrm{~mm}$ posterior to bregma, (ML) $\pm 1 \mathrm{~mm}$, (DV) $-1.2 \mathrm{~mm}$ from the skull, and for the CA3 infusion site as follows: (AP) $-1.6 \mathrm{~mm}$, (ML) $\pm 2.5 \mathrm{~mm}$, (DV) $-1.5 \mathrm{~mm}$, according to the brain atlas of Franklin and Paxinos (1997). Dental cement (polycarboxylate, Sigma) was used to fasten the guide cannulae to the skull. Stainless-steel obturators were inserted into guide cannulae to prevent occlusion and left in place until the injections were made. A total of 111 male mice were operated on and tested. After surgery, mice were allowed at least $1 \mathrm{wk}$ to

Figure 8. Effects of CA3 and CA1 pre-test infusions of lidocaine on the retrieval of contextual fear (Experiment 3). (A) Contextual fear; CA3- and CA1-infusions of lidocaine $10 \mathrm{~min}$ testing failed to impair the contextual fear retrieval in regard to $\mathrm{NaCl}$ control $(P=0.271)$. (B) Kinetics of contextual freezing. Hippocampal CA3 and CA1 lidocaine infusions failed to impair the tempora evolution of freezing, suggesting that the recall of contextual temporal learning is independent of these two areas. (C) No significant effect of pre-test drug infusions is revealed by the conditioning to the tone $(P=0.847)$, confirming its independence from the CA 3 and CA1 areas.

normally through recognition memory when the neural activity of these two hippocampal subregions is blocked. In this study, we demonstrated the involvement of the CA3 region in forming configural representations. Its recurrent network has been suggested to provide a mechanism for maintaining coherent information for short-term duration and to serve as a temporary buffer for episodic and working memories through reverberating activity in the connections of the recurrent collaterals (Kesner and Rolls 2001). It is also considered as a critical region for cognitive functions related to memory recall through pattern completion (Nakazawa et al. 2002). Our results indicate that the CA3 region is necessary for both optimal encoding and consolidation of contextual memory, whereas in our experimental conditions, this area is neither involved in context recognition nor in tone memory processing.

Moreover, our study emphasizes the particular role of the CA1 area (1) in memory consolidation and, (2) as suggested by the analysis of contextual freezing kinetics, its possible involvement in processing the temporal component of memory (the "when") in addition to its implication in the acquisition and consolidation of the representation of the context (the "where"). These results complete previous knowledge on functional specificity of hippocampal subregions that assumes the involvement of the CA3 area in spatial and temporal working memory in pattern completion and in pattern association, with CA1 being more important in temporal pattern separation (Kesner et al. 2000).

In summary, our results suggest that the CA3 network, via prior information processing in the dentate gyrus, could support acquisition and also consolidation of the association of incoming multisensori-information patterns in order to build a unified representation of the context, whereas the CA1 area would be more important for memory consolidation and might also deal with the temporal component of the contextual representation.

\section{Materials and Methods}

\section{Subjects}

The subjects were C57BL/6J 9-12-wk-old male mice obtained from Charles River and reared in the CRCA breeding facility. They were housed in groups of from 3 to 5 per cage and maintained at a constant temperature $\left(21 \pm 1{ }^{\circ} \mathrm{C}\right)$ with a 12-h light/ 12-h dark cycle (lights on at 8:00 a.m.). Water and food were available ad libitum.

\section{Implantation of guide cannulae} for intrahippocampal microinfusions

Mice were anaesthetized with a mixture of ketamine hydrochloride (100 mg/kg, i.p.) (Vribac) and xylazine (15 mg/kg, i.p.) (Ramrecover, and were gently handled daily by the experimenter to
minimize the stress associated with handling throughout the experiments. All experiments were carried out in the afternoon during the diurnal phase. This work was carried out in accordance with the Policies of the French Committee of Ethics. S. Daumas, B. Francés, and J-M. Lassalle are authorized by the French Direction of Veterinary Services to conduct surgery and behavioral experiments on vertebrates (Authorization \#31-111, \#03-817, and \#31-122).

\section{Intrahippocampal microinfusion procedure and drug diffusion}

On test days, the animals were carried to the surgery room, where injections were made. Prior to each injection, the obturators were removed and an infusion cannula was inserted extending 1.1 $\mathrm{mm}$ beyond the end of the guide cannula for CA3 infusions and $0.9 \mathrm{~mm}$ for CA1 infusions. The infusion cannula was connected by a polypropylene tube to a Hamilton microsyringe that delivered the solution at the rate of $0.11 \mu \mathrm{L} / \mathrm{min}$, using an automated pump. A volume of $0.25 \mu \mathrm{L}$ of a $2 \%$ lidocaine hydrochloride solution (Sigma) or saline was infused into each dorsal hippocampus. After completing the infusion, the injection cannulae were left in place for an additional $60 \mathrm{sec}$. Lidocaine is an amidelinked local anesthetic that reduces sodium conduction by blocking voltage-gated sodium channels, thereby preventing membrane depolarization and conduction of the action potential. We used lidocaine for its short duration effect. Tehovnik and Sommer (1997) have demonstrated that monkey cerebral cortex units were inactivated $<8 \mathrm{~min}$ after intracortical lidocaine injection, and that they gradually recovered, regaining much of their initial activity by $30 \mathrm{~min}$ after the beginning of the injection. They could estimate the volume of lidocaine required to inactivate $>90 \%$ neurons using the spherical volume equation, $V=4 / 3 \pi R^{3}$. Martin (1991) has demonstrated, using autoradiography on brain slices, that $1 \mu \mathrm{L}$ of lidocaine spreads in a radial fashion to a distance of $1.7 \mathrm{~mm}$ from the site of infusion in both cortical and subcortical tissues, and that its peak activity occurs $10 \mathrm{~min}$ postinfusion. In regard to these data and unpublished experiments made in our laboratory studying the diffusion of a dye, the chosen volume of the bolus $(0.25 \mu \mathrm{L})$ ensures that lidocaine spreads in, and by consequence, inactivates only the studied area (CA3 or CA1) as illustrated in Figure 1. Depending on the experimental group, the animals were injected before conditioning (15 or 10 min before), immediately after conditioning, or $10 \mathrm{~min}$ before memory testing.

\section{Apparatus for behavioral testing}

Conditioning took place in a conditioning chamber that consisted of a rectangular polyvinyl chloride box (length $35 \mathrm{~cm}$, width $20 \mathrm{~cm}$, and height $25 \mathrm{~cm}$ ) with three light-brown sides and a Plexiglas front wall, through which experimental subjects were videotaped. The floor was made of a grid with stainless-steel rods (diameter $4 \mathrm{~mm}$ ) spaced $1 \mathrm{~cm}$ and connected to a generator 
(Campden Instruments) delivering shocks of defined duration (2 sec) and intensity $(0.7 \mathrm{~mA})$ through a shock-scrambler unit. Light-brown disposable tissue paper covered the floor below the grid. The loudspeaker producing the tone $(85 \mathrm{~dB}, 30 \mathrm{sec})$ was fixed on the top of the conditioning chamber. The experimental device, lit by a $60 \mathrm{~W}$ white bulb was surrounded by a white curtain. Two black and white patterns faced the conditioning chamber. Experiments were recorded using a video camera placed in front of the conditioning chamber, connected to a TV monitor and a video tape recorder placed in the adjacent room, where the experimenter and all of the electronic system were settled. The conditioning chamber was cleaned with 70\% aqueous ethanol before each conditioning session. Contextual learning was checked in the same experimental conditions as conditioning, whereas tone learning was assessed in a modified context. For that purpose, the external patterns were removed. The modified chamber was made triangular by adjunction of white Plexiglas walls and floor. The apparatus was washed with $1 \%$ acetic acid and lit by a $40 \mathrm{~W}$ white bulb.

\section{Contextual fear-conditioning procedure}

Behavioral testing started $1 \mathrm{wk}$ after surgery. Conditioning consisted of a single conditioning session with two trials. During conditioning, mice stayed in the conditioning chamber for a total of $5 \mathrm{~min}, 30 \mathrm{sec}$. The mouse was dropped by the experimenter into the conditioning chamber via the ceiling. After a 2-min exploration period, a sound (CS) was emitted for $30 \mathrm{sec}$, and a foot shock (US) was superposed to the tone during the last $2 \mathrm{sec}$. After an intertrial interval of $2 \mathrm{~min}$, the paired CS-US was repeated, and $30 \mathrm{sec}$ after the second foot-shock, mice were gently removed from the chamber and returned to their home cage. Twenty four hours after conditioning, mice were individually checked for freezing to the context in the conditioning chamber for 4 min (memory testing). Two hours later, they were tested for freezing to the tone in the modified context; 2 min after their introduction in the modified chamber, mice received a 2-min tone presentation.

\section{Activity and freezing measurements}

In order to ensure that drugs do not act directly on mobility, locomotor activity of mice was also measured during the first 2 min of conditioning (Anagnostaras et al. 1999; Lee and Kesner 2004a). It was defined as the number of crossings of a virtual line dividing the conditioning chamber in two parts. Freezing is defined as the lack of movement beside respiration and heartbeats. Freezing was scored every $5 \mathrm{sec}$ during conditioning and test sessions. The data were converted to the percentage of samples scored at freezing and calculated for the 4-min context test period, the 2-min pre-tone, and the 2-min tone test presentation.

\section{Histological examination of cannulae tips and infusions locations}

At the end of behavioral experiments, all mice were killed with an overdose of chloral hydrate $(800 \mathrm{mg} / \mathrm{kg}$, i.p.) and their brains removed. Brains were stored $24 \mathrm{~h}$ in a solution of $2.5 \%$ glutaraldehyde-30\% sucrose $(1: 1)$ to fix the tissue; then, they were placed for $24 \mathrm{~h}$ in sucrose (30\%) and stored in a refrigerator for dehydration. Afterward, brains were cut into $40-\mu \mathrm{m}$ coronal sections with a freezing microtome. To verify that brains had been infused to the correct site, sections were mounted on gelatintreated slides and stained with thionin. After staining, sections were dehydrated through alcohol series, cleared with toluene, and cover-slipped with neoentellan (Mérux). The sections, only identified by noninformative numbers, were then examined with a light microscope to verify the good placement of guide cannulae and of infusion sites.

\section{Data analysis}

Mean freezing percentages for each group ( \pm SEM) are presented in the figures. Group sizes ranged from seven to nine mice. To satisfy the requirements for the use of ANOVA, the mean per- centages of freezing scores $(\mathrm{P})$ were transformed in $\mathrm{Q}=\arcsin (\sqrt{\mathrm{P}} /$ 100). Statistical analyses were performed on the $Q$ variable, using one-way analysis of variance (ANOVA), or repeated measures ANOVA design for related samples (SYSTAT 9 for windows). All post-hoc comparisons were conducted using Fisher's LSD test. $\alpha$ Levels were set at $P<0.05$ for all tests.

\section{Acknowledgments}

We thank Doctor Claire Rampon for critically reading the manuscript. This study was supported by fundings from CNRS, Université Paul Sabatier and French Department of Research (Action Concertée Incitative "Neurosciences Intégratives et Computationnelles").

\section{References}

Anagnostaras, S.G., Maren, S., and Fanselow, M.S. 1999. Temporally graded retrograde amnesia of contextual fear after hippocampal damage in rats: Within-subjects examination. J. Neurosci. 19: $1106-1114$

Anagnostaras, S.G., Gale, G.D., and Fanselow, M.S. 2001. Hippocampus and contextual fear conditioning: Recent controversies and advances. Hippocampus 11: 8-17.

Bast, T. and Feldon, J. 2003. Hippocampal modulation of sensorimotor processes. Progress in Neurobiol. 70: 319-345.

Brun, V.H., Otanaess, M.K., Molden, S., Steffenach, H.-A., Witter, M.P., Moser, M.-B., and Moser, E.I. 2002. Place cells and place recognition maintained by direct entorhinal-hippocampal circuitry. Science 296: $2243-2246$.

Eichenbaum, H. 1996. Is the rodent hippocampus just for 'place'? Curr. Opin. Neurobiol. 6: 187-195.

Fanselow, M.S. 2000. Contextual fear, gestalt memories, and the hippocampus. Behav. Brain Res. 110: 73-81.

Fortin, N.J., Agster, K.L., and Eichenbaum, H.B. 2002. Critical role of the hippocampus in memory for sequences of events. Nat. Neurosci. 5: 458-462.

Forwood, S.E., Winters, B.D., and Bussey, T.J. 2005. Hippocampal lesions that abolish spatial maze performance spare object recognition memory at delays up to 48 hours. Hippocampus 19: 347-355.

Franklin, K.B.J. and Paxinos, G. 1997. The mouse brain in stereotaxic coordinates. Academic Press, San Diego, CA.

Gerlai, R. 1998. Contextual learning and cue association in fear conditioning in mice: A strain comparison and a lesion study. Behav. Brain Res. 95: 191-203.

Huerta, P.T., Sun, L.D., Wilson, M.A., and Tonegawa, S. 2000. Formation of temporal memory requires NMDA receptors within CA1 pyramidal neurons. Neuron 25: 473-480.

Ji, J.Z., Wang, X.M., and Li, B.M. 2003. Deficit in long-term contextual fear memory induced by blockade of beta-adrenoceptors in hippocampal CA1 region. Eur. J. Neurosci. 17: 1947-1952.

Kandel, E.R. 2001. The molecular biology of memory storage: A dialogue between genes and synapses. Science 294: 1030-1038.

Karlsson, T., Johansson, I., Adolsson, R., Nilsson, L.-G., and Dubuc, S. 2003. Recognition memory in Alzheimer's disease: A demonstration of a remarkable memory capacity in Alzheimer's disease. Dement. Geriat. Cog. 15: 6-9.

Kesner, R.P. and Rolls, E.T. 2001. Role of long-term synaptic modification in short-term memory. Hippocampus 11: 240-250.

Kesner, R.P., Gilbert, P.E., and Wallenstein, G.V. 2000. Testing neural network models of memory with behavioral experiments. Curr. Opin. Neurobiol. 10: 260-265.

Kim, J.J. and Fanselow, M.S. 1992. Modality-specific retrograde amnesia of fear. Science 256: 675-677.

Kim, J.J., Fanselow, M.S., DeCola, J.P., and Landeira-Fernandez, J. 1992. Selective impairment of long-term but not short-term conditional fear by the N-methyl-D-aspartate antagonist APV. Behav. Neurosci. 106: $591-596$

Kjelstrup, K.G., Tuvnes, F.A., Steffenach, H.-A., Murison, R., Moser, E.I., and Moser, M.-B. 2002. Reduced fear expression after lesions of the ventral hippocampus. Proc. Natl. Acad. Sci. 99: 10825-10830.

Lee, I. and Kesner, R.P. 2002. Differential contribution of NMDA receptors in hippocampal subregions to spatial working memory. Nat. Neurosci. 5: 162-168.

. 2004a. Differential contributions of dorsal hippocampal subregions to memory acquisition and retrieval in contextual fear-conditioning. Hippocampus 14: 301-310. . 2004b. Encoding versus retrieval of spatial memory: Double dissociation between the dentate gyrus and the perforant path inputs into CA3 in the dorsal hippocampus. Hippocampus 14: 66-76. 
Lee, I., Rao, G., and Knierim, J.J. 2004. A double dissociation between hippocampal subfields: Differential time course of CA3 and CA1 place cells for processing changed environments. Neuron 42: $803-815$.

Martin, J.H. 1991. Autoradiographic estimation of the extent of reversible inactivation produced by microinjection of lidocaine and muscimol in the rat. Neurosci. Lett. 127: 160-164.

Mayes, A.R., Holdstock, J.S., Isaac, C.L., Montaldi, D., Grigor, J., Gummer, A., Cariga, P., Downes, J.J., Tsivilis, D., Gaffan, D., et al 2004. Associative recognition in a patient with selective hippocampal lesions and relatively normal item recognition. Hippocampus 14: 763-784.

McClelland, J.L., McNaughton, B.L., and O'Reilly, R.C. 1995. Why there are complementary learning systems in the hippocampus and neocortex: Insights from the successes and failures of connectionist models of learning and memory. Psychol. Rev. 102: 419-457.

McGaugh, J.L. 2000. Memory-A century of consolidation. Science 287: 248-251.

McIntosh, A., Sekuler, A.B., Penpeci, C., Rajah, M., Grady, C., Sekuler, R., and Bennet, P. 1999. Recruitment of unique neural systems to support visual memory in normal aging. Curr. Biol. 9: 1275-1278.

Morris, R.G. 2001. Episodic-like memory in animals: Psychological criteria, neural mechanisms and the value of episodic-like tasks to investigate animal models of neurodegenerative disease. Phil. Trans. R. Soc. Lond. B. 356: 1453-1465.

Morris, R.G., Moser, E.I., Riedel, G., Martin, S.J., Sandin, J., Day, M., and O'Carroll, C. 2003. Elements of a neurobiological theory of the hippocampus: The role of activity-dependent synaptic plasticity in memory. Philos. Trans. R. Soc. Lond. B Biol. Sci. 358: 773-786.

Moser, M.B. and Moser, E.I. 1998. Functional differentiation in the hippocampus. Hippocampus 8: 608-619.

Moser, E., Moser, M.B., and Andersen, P. 1993. Spatial learning impairment parallels the magnitude of dorsal hippocampal lesions, but is hardly present following ventral lesions. J. Neurosci. 13: 3916-3925.

Moser, M.B., Moser, E.I., Forrest, E., Andersen, P., and Morris, R.G. 1995. Spatial learning with a minislab in the dorsal hippocampus. Proc. Natl. Acad. Sci. 92: 9697-9701.

Nakazawa, K., Quirk, M.C., Chitwood, R.A., Watanabe, M., Yeckel, M.F., Sun, L.D., Kato, A., Carr, C.A., Johnston, D., Wilson, M.A., et al. 2002. Requirement for hippocampal CA3 NMDA receptors in associative memory recall. Science 297: 211-218.

Nakazawa, K., Sun, L.D., Quirk, M.C., Rondi-Reig, L., Wilson, M.A., and Tonegawa, S. 2003. Hippocampal CA3 NMDA receptors are crucial for memory acquisition of one-time experience. Neuron 38: 305-315. Nakazawa, K., McHugh, T.J., Wilson, M.A., and Tonegawa, S. 2004. NMDA receptors, place cells and hippocampal spatial memory. Nat. Rev. Neurosci. 5: 361-372.

Phillips, R.G. and LeDoux, J.E. 1992. Differential contribution of amygdala and hippocampus to cued and contextual fear conditioning. Behav. Neurosci. 106: 274-285.

Riedel, G., Casabona, G., Platt, B., Macphail, E.M., and Nicoletti, F. 2000. Fear conditioning-induced time- and subregion-specific increase in expression of mGlu5 receptor protein in rat hippocampus. Neuropharmacology 39: 1943-1951.

Rolls, E.T. and Treves, A. 1996. The hippocampus and memory. In: Neural networks and brain function, pp. 95-135. Oxford University Press.

Rudy, J.W. and O'Reilly, R.C. 2001. Conjunctive representations, the hippocampus, and contextual fear conditioning. Cognit. Affect. Behav. Neurosci. 1: 66-82.

Sekuler, R., Kahana, M.J., McLaughlin, C., Golomb, J., and Wingfield, A. 2005. Preservation of episodic visual recognition memory in aging. Exper. Aging Res. 3: 11-13.

Shimizu, E., Tang, Y.P., Rampon, C., and Tsien, J.Z. 2000. NMDA receptor-dependent synaptic reinforcement as a crucial process for memory consolidation. Science 290: 1170-1174.

Squire, L.R. 1992. Memory and the hippocampus: A synthesis from findings with rats, monkeys, and humans. Psychol. Rev. 99: 195-231.

Squire, L.R., Schmolck, H., and Starck, S.M. 2001. Impaired auditory memory in amnesic patients with medial temporal lobe lesions. Learn. Mem. 8: 252-256.

Tehovnik, E.J. and Sommer, M.A. 1997. Effective spread and timecourse of neural inactivation caused by lidocaine injection in monkey cerebral cortex. J. Neurosci. Methods 74: 17-26.

Tsien, J.Z., Huerta, P.T., and Tonegawa, S. 1996. The essential role of hippocampal CA1 NMDA receptor-dependent synaptic plasticity in spatial memory. Cell 87: 1327-1338.

Tulving, E. 1983. Elements of episodic memory. Oxford University Press, New York.

Zhang, W.N., Bast, T., and Feldon, J. 2002. Effects of hippocampal $\mathrm{N}$-methyl-D-aspartate infusion on locomotor activity and prepulse inhibition: Differences between the dorsal and ventral hippocampus. Behav. Neurosci. 116: 72-84.

Received May 20, 2004; accepted in revised form April 26, 2005. 


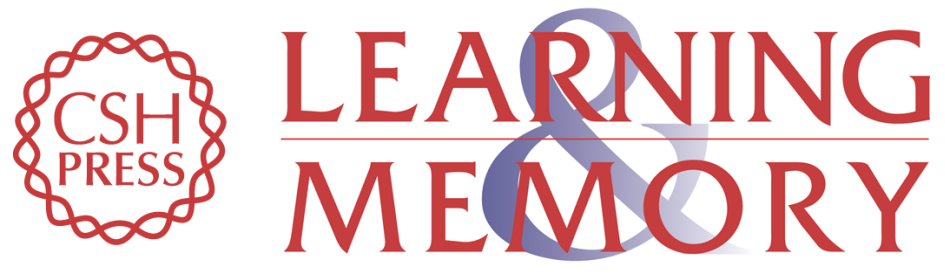

\section{Encoding, consolidation, and retrieval of contextual memory: Differential involvement of dorsal CA3 and CA1 hippocampal subregions}

Stéphanie Daumas, Hélène Halley, Bernard Francés, et al.

Learn. Mem. 2005, 12:

Access the most recent version at doi:10.1101/lm.81905

References This article cites 44 articles, 11 of which can be accessed free at: http://learnmem.cshlp.org/content/12/4/375.full.html\#ref-list-1

License

Email Alerting

Receive free email alerts when new articles cite this article - sign up in the box at the Service top right corner of the article or click here. 